\title{
A patient with a traumatic brain injury due to barrel bomb tertiary blast effect
}

\author{
Mustafa Bolatkale1, Çağdaş Can², Ahmet Çağdaş Acara ${ }^{3 *}$, Aydın Sarıhan ${ }^{4}$ \\ 'Medipol University Hospital, Istanbul, Turkey \\ ${ }^{2}$ Merkezefendi State Hospital, Manisa, Turkey \\ ${ }^{3}$ Gaziemir State Hospital, İzmir, Turkey \\ ${ }^{4}$ Manisa State Hospital, Manisa, Turkey
}

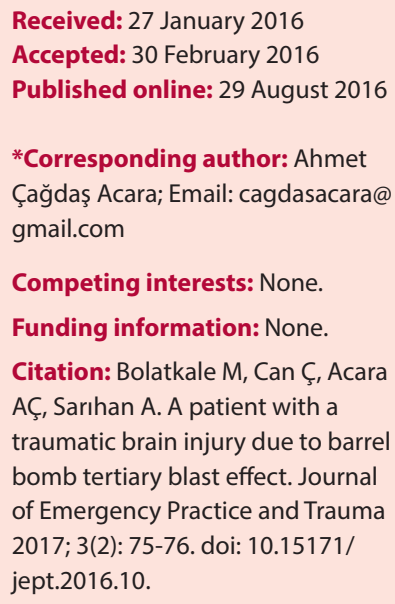

\begin{abstract}
Preparing to manage weapons of mass destruction events challenges emergency services systems neighboring Syria every day. Understanding injury from explosives is essential for all providers of emergency care in both civilian and military settings. In this case, the authors present a 22-year-old man who was admitted to the emergency department with displaced skull fracture, epidural hemorrhage and cerebral contusion due to barrel bomb tertiary blast effect. A 22-year-old man who complained of pain in the right temporal head region after barrel bomb explosion was admitted in the emergency department. The patient could not remember the explosion and found himself on the ground. In his medical history, there was not a record of any diseases, operations or traumas. Examination of the head revealed scalp hematoma and slump in the skull on the right temporal region. Patients computed tomography (CT) scan showed a displaced skull fracture, epidural hematoma and cerebral contusion.

Keywords: Barrel bomb, Blast effect, Head trauma
\end{abstract}

\section{Introduction}

The barrel bomb is essentially a large, home-made incendiary device. An oil barrel or a similar cylindrical container filled with petrol or other crude shrapnel, along with explosives can produce devastating casualties in small and large numbers (1). Barrel bombs create an immediate rise in pressure, making a shock wave that travels outward at supersonic speed which is called blast effect (2). Explosives or blast injuries are considerable clinical issues in military medicine, with a significant number of casualties being injured by improvised explosive devices in the current conflicts in Iraq and Afghanistan (1). Injuries occur as a direct result of blast wave-induced changes in atmospheric pressure (primary blast injury), from objects put in motion by the blast hitting people (secondary blast injury) and by people being forcefully put in motion by the blast (tertiary blast injury) (3). This patient was driven away for about four meters and harshly hit his head on the ground with blast effect of the explosion. It is interesting that such an explosion with its blast effect cannot be the reason of tympanic membrane rupture, pneumothorax or hollow internal organ perforation. Why such an explosion can cause head injury in this patient? This situation can be due to the bomb tertiary blast effect. We believe that bomb tertiary blast effect might prevent explosion associ- ated severe injuries by moving away the patient from the danger zone. But it is important to notice that blast waves can cause damage to the brain tissue by itself without any traumatic processes (2).

\section{Case Presentation}

A 22-year-old man who complained of pain in the right temporal head region after barrel bomb explosion was admitted in the emergency department. The patient could not remember the explosion and found himself on the ground by the blast effect of the bomb. In his medical history, there was not a record of any diseases, operations or traumas. He had no drug allergies, did not drink alcohol or smoke. He did not take any drugs. On emergency department admission, the patient's vital signs were normal. Examination of neurologic and other systems were normal except traumatic amnesia. His medical history was unremarkable. Examination of the head revealed scalp hematoma and slump in the skull on the right temporal region.

\section{Conclusion}

Patient's computed tomography (CT) scan showed a displaced skull fracture, epidural hematoma and cerebral contusion (Figures 1 and 2). He was admitted to the neu- 


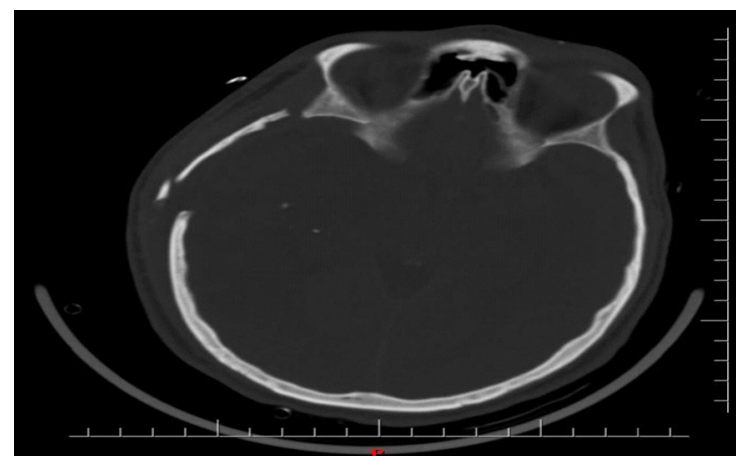

Figure 1. Displaced skull fracture.

rosurgery department for follow up, and after 10 days of follow up he was discharged from hospital with full recovery.

When compared with patients injured via other types of trauma (gunshot wound), victims of terrorist bombs are primarily in the age range of 15-44, have more severe injuries, require more surgical intervention, and have three times the rate of mortality (4).

It is important that emergency care providers understand the nature of the explosive weapons that are causing death and serious injuries with various mechanisms.

\section{Ethical issues}

This study was carried out in accordance with ethical guidelines and WMA Declaration of Helsinki.

\section{Authors' contributions}

All authors contributed to the intellectual planning of the

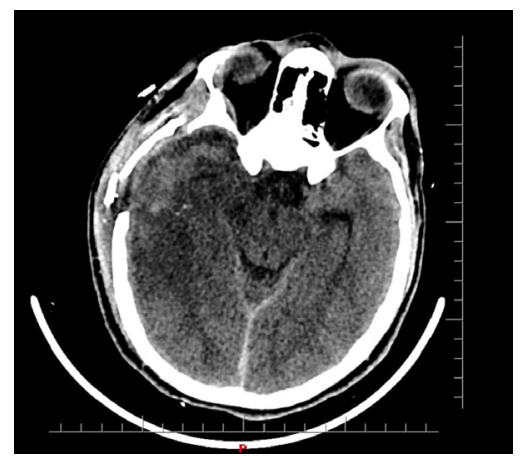

Figure 2. Epidural hematoma and cerebral contusion

project, intellectual analysis of the data, and writing of the paper.

\section{References}

1. Frykberg ER. Medical management of disaster and mass casualties from terrorist bombings: How can we cope? J Trauma 2002;53(2):201-12. doi: 10.1097/01. TA.0000021586.40033.BA.

2. Kluger Y, Peleg K, Daniel-Aharonson L, Mayo A, Israeli Trauma Group. The special injury pattern in terrorist bombings. J Am Coll Surg 2004;199(6):875-9. doi: 10.1016/j. jamcollsurg.2004.09.003.

3. Taber KH, Warden DL, Hurley RA. Blast-Related Traumatic Brain Injury: What Is Known? J Neuropsychiatry Clin Neurosci 2006;18(2):141-5. doi: 10.1176/jnp.2006.18.2.141.

4. Frykberg ER, TepasJJ 3rd. Terrorist bobmbings. Lesson learned from Belfast to Beriut. Ann Surg 1988; 208(5): 56976. 